\title{
DIMENSIO NAMIENTO DE UN INTERCAMBIADOR DE CALOR DE TUBO Y CORAZA PARA FLUIDOS NO NEWTONIANOS POR LOS METODOS DE KERN Y TINKER
}

\section{A DESIGN OF A TUBE AND SHELL HEAT EXCHANGER FOR NON-NEWTONIANOS FLUIDES BASED ON KERN AND TINKER METHODS}

\author{
Everaldo J. Montes ${ }^{1}$, Fabián A. O rtega ${ }^{1}$, Ricardo D. Andrade ${ }^{1}$ y Alba M. Durango ${ }^{1}$
}

Recibido para evaluación: Marzo 2006

Aceptado para publicación: Junio 2006

\begin{abstract}
RESU MEN
Los intercambiadores de calor de tubo y coraza son utilizados en las industrias de alimentos, químicas y farmacéuticas. Fue dimensionado un intercambiador de calor para enfriar jugo de naranja con concentración de $27^{\circ} \mathrm{Brix}$, utilizando los métodos de Kern y Tinker que son los más conocidos y disponibles en la literatura. Los resultados muestran que existen discrepancias entre los dos métodos hasta $300 \%$ en el dimensionamiento hidráulico y del $70 \%$ en el coeficiente de transferencia de calor convectivo.
\end{abstract}

Palabras claves: Transferencia de calor, coraza, flujo laminar, pérdida de carga.

\begin{abstract}
Shell and tube heat exchangers are used in food, chemical and pharmaceutical industries. A heat exchanger was projected for heating orange juice with $27^{\circ} \mathrm{Brix}$. The methods Kern and Tinker were applied because they are the most known and available in the literature. The results show the discrepancies between the two methods up to $300 \%$ of divergences in the hydraulic project and $70 \%$ in the convection coefficient of heat transfer.
\end{abstract}

Key Words: Transfer, shield, laminar flow, charge los

${ }^{1}$ Universidad de Córdoba. Departamento de Ingeniería de Alimentos, Km. 12 vía Cereté - Ciénaga de O ro Tel. (4) 8940508 - Fax 7860255 Email: emontes@ sinu.unicordoba.edu.co 


\section{INTRODUCCION}

Las Industrias de fluídos alimenticios como las de jugo integral y concentrado, café soluble, leche condensada, azúcar, etc., utilizan equipos para intercambio de calor, encontrándose una amplia variedad de tipos de intercambiadores de calor con diversos niveles de complejidad tecnológica como preenfriadores, enfriadores y condensadores.

Estos equipos requieren grandes áreas para la transmisión de calor, que pueden ser obtenidas por un equipo multitubular que consiste en diversos tubos montados paralelamente en el interior de una carcaza o coraza, los cuales son denominados intercambiadores de calor de tubo y coraza (Geankoplis, 1993).

Los intercambiadores son ampliamente usados y fabricados en muchos tamaños, con varios arreglos de flujo y de diversos tipos; pueden operar en grandes caídas de presión y temperatura. La facilidad de fabricación y el costo relativamente bajo constituyen la principal razón para su empleo ilimitado en las aplicaciones de ingeniería (O zisik, 1990).

El Proyecto de un intercambiador de calor es un asunto complejo: la transferencia de calor, la pérdida de carga, el dimensionamiento, la evaluación del desempeño y los aspectos económicos juegan papeles muy importantes en el resultado final. La construcción de un intercambiador de calor puede ser dividido en tres fases principales: análisis térmico, proyecto mecánico preliminar y fabricación (Spano, 1961).

El análisis térmico puede ser determinado por varios métodos, algunos disponibles en la literatura técnica común y otros muy mejorados, generalmente acoplados a los programas de computador, que pueden ser obtenidos por medio de contratos con entidades privadas especializadas en equipos de intercambio térmico, tales como: HTRI, Heat Transfer Research Inc, en los Estados Unidos y HTFS, Heat Transfer and Fluid Flow Service, en Inglaterra.

Entre los principales métodos de análisis térmicos encontrados en la literatura común, están los de Kern y Tinker. El primero es el más tradicional, pero acumula mayor número de errores, principalmente en el flujo del lado de la coraza, en las pérdidas de carga y en el flujo laminar. Por otro lado, el segundo, conocido por el análisis de las corrientes, es considerado el más completo, sirviendo de soporte para los métodos más desarrollados (O zisik, 1990; Kern, 1999).

Tinker plantea que la clave para la solución del flujo a través de la coraza, consiste en dividirlo en una serie de corrientes separadas. La Figura 1 muestra las corrientes A, B, C y D, que influyen de manera distinta en cuanto a la transferencia de calor y pérdida de carga para el lado de coraza (O sizik, 1990).
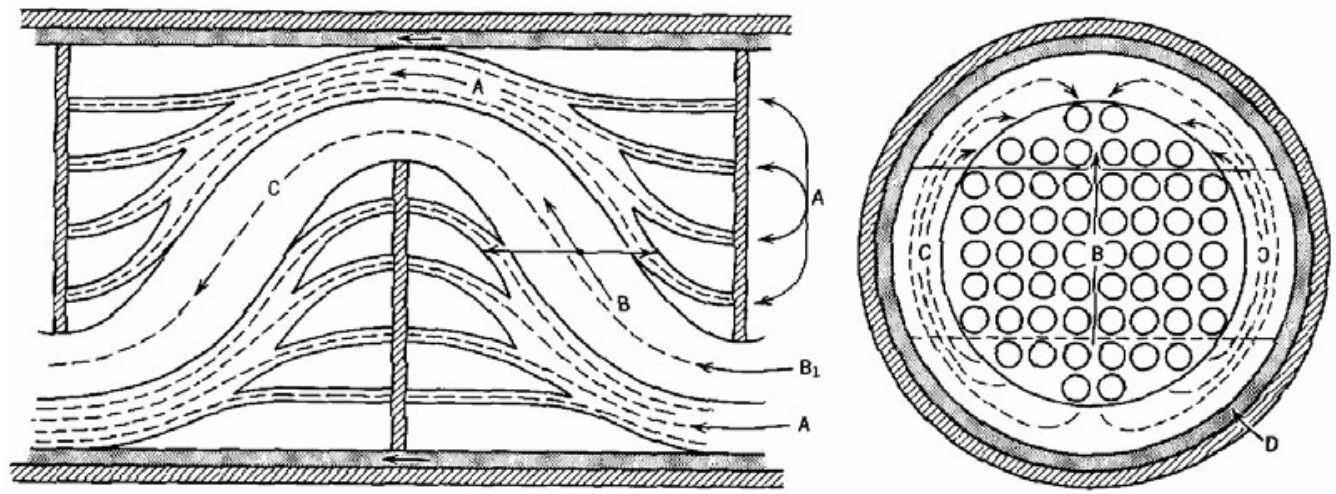

Figura 1. Flujo dividido en corrientes de Tinker

Fuente: O zisik (1990). 
En el presente trabajo se dimensiona un intercambiador de calor de tubo y coraza para jugo de naranja (fluido no-Newtoniano), por los métodos de Kern y Tinker, comparánd olos en cuanto a las condiciones de flujo desarrollado, lo cual es importante para evitar un sub o super dimensionamiento del equipo, pudiendo generar desde gastos excesivos de energía e inversión inicial, hasta daños en el producto. La diferencia entre los dos métodos será ilustrada a partir de los resultados obtenidos.

\section{MATERIALES Y METODOS}

\section{Propiedades físicas del fluido}

El conocimiento de las propiedades físicas de los fluidos en movimiento es muy importante para el proyecto termohidráulico del equipo.

Como fluido principal del intercambiador, fue utilizado jugo de naranja concentrado de $27^{\circ} \mathrm{Brix}$ y temperatura variando entre 50 y $60^{\circ} \mathrm{C}$; tal fluido presenta comportamiento plástico Casson. Sus propiedades físicas fueron obtenidas de los trabajos de Telis- Romero et al. (1998; 1999).

\section{Dimensionamiento del intercambiador de calor}

El dimensionamiento de un intercambiador de calor es dividido en dosfases: transferencia de calor y pérdida de carga.

\section{Nomenclatura}

\begin{tabular}{|c|c|}
\hline $\mathrm{m}$ & Viscosidad del agua \\
\hline$t_{0}$ & Tensión inicial de flujo \\
\hline 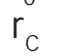 & Densidad del agua \\
\hline & Densidad del jugo \\
\hline & $\begin{array}{l}\text { Viscosidad del agua en la temperatura } \\
\text { de la pared }\end{array}$ \\
\hline & Caída de presión en la coraza \\
\hline & Caída de presión en los retornos \\
\hline & Caída de presión total en los tubos \\
\hline & en los tubos \\
\hline
\end{tabular}

A : Area de intercambio

$A_{C} \quad$ : Area de flujo de la coraza

$A_{i} \quad$ : Area de flujo de los tubos

B : Número de deflectores

$\mathrm{C}_{\mathrm{m}}$ : (número de tubos transversales / número de hileras de tubos) $)^{0,5}$

$C_{p}:$ : Calor específico del agua

$c_{p} \quad$ : Calor específico del jugo

d : Diámetro externo de los tubos

$D_{c} \quad$ : Diámetro interno de coraza

D : Diámetro equivalente de la coraza

$\mathrm{d}_{\mathrm{i}}$ : Diámetro interno de los tubos

$\mathrm{f}_{\mathrm{c}} \quad$ : Factor de fricción de Darcy para la coraza

$\mathrm{F}_{\mathrm{h}}, \mathrm{M}_{1}$ : Factores de corrección de Tinker para transferencia de calor en la coraza

$\mathrm{f}_{\mathrm{i}} \quad$ : Factor de fricción de Darcy para los tubos

$\mathrm{G}_{c}$ : Flujo másico del agua

$\mathrm{G}_{\mathrm{i}} \quad$ : Flujo másico del jugo

$\mathrm{H}$ : Altura de la chicana

$\mathrm{h}_{\mathrm{c}}$ : Coeficiente de transferencia de calor convectivo en la coraza

$h_{i} \quad$ : Coeficiente de transferencia de calor convectivo en el interior de los tubos

$\mathrm{K}_{\mathrm{b}} \quad$ : Indice de consistencia medio

$\mathrm{k}_{\mathrm{c}} \quad$ : Conductividad térmica del agua

$\mathrm{k}_{\mathrm{i}} \quad$ : Conductividad térmica del jugo

$\mathrm{K}_{\mathrm{p}} \quad$ : Indice de consistencia en la temperatura de la pared

L : : Longitud de los tubos

M : Tasa másica de agua

m : Tasa másica de jugo de naranja

$\mathrm{n} \quad$ : Indice de comportamiento del jugo

$\mathrm{N} \quad$ : Número de pasos de los tubos

Q : Calor intercambiado

$\mathrm{R}_{d} \mathrm{Re}_{c}$ : Resistencia a la transferencia de calor Reynolds para los tubos

$\operatorname{Re}_{h}$ : Reynolds modificado para la transferencia de calor en la coraza

$\operatorname{Re}_{p} \quad$ : Reynolds modificado para caída de presión en la coraza

$\mathrm{S}$ : Pitch

$t_{1} \quad$ : Temperatura de entrada de el jugo de naranja

$\mathrm{T}_{1} \quad$ : Temperatura de entrada del agua

$t_{2}$ : Temperatura de salida del jugo de naranja

$\mathrm{T}_{2} \quad$ : Temperatura de salida del agua 
$U_{c} \quad$ : Coeficiente global de transferencia de calor limpio

$U_{D} \quad$ : Coeficiente global de transferencia de calor del proyecto

$X \quad$ : Número de tubos

$Y, F_{p}$ : Factores de corrección de Tinker para caída de presión en la coraza

\section{Cálculo de la transferencia de calor}

La modificación de la ecuación general de Fourier, integrada para un flujo permanente puede ser igualada a un balance entálpico y escrita en la siguiente forma:

$\mathrm{Q}=\mathrm{U}_{\mathrm{D}} \cdot \mathrm{A} \cdot \Delta \mathrm{t}=\mathrm{MC}_{\mathrm{P}}\left(\mathrm{T}_{1}-\mathrm{T}_{2}\right)=\mathrm{mc}_{\mathrm{P}}\left(\mathrm{t}_{2}-\mathrm{t}_{1}\right)$

Como el intercambiador propuesto tendrá flujo tanto en contracorriente como en paralelo, la verdadera diferencia de temperatura (Dt) utilizada por Kern es una modificación de la deducción de Underwood (Kern, 1999).

El cálculo de la transferencia de calor se divide en dos partes, para el lado de la carcaza y para el interior de los tubos. Para el interior de los tubos el coeficiente de transmisión de calor convectivo está dado por la ecuación 2 de Metzner y Gluck (Geankoplis, 1993)

$\frac{h_{i} d_{i}}{k_{i}}=1,75\left(\frac{3 n+1}{4 n}\right)\left(\frac{m c_{p}}{k_{i} L}\right)^{1 / 3}\left(\frac{K_{b}}{K_{p}}\right)^{0,14}$

Para el lado de la carcaza los métodos de Kern y Tinker se diferencian en (O sizik, 1990):

Kern, utiliza en los cálculos del número de Reynolds, el diámetro equivalente para un flujo radial, correlacionándolo experimentalmente con el coeficiente de transmisión de calor convectivo, en un diagrama, a través de la ecuación 3:

$\frac{h_{c} D_{e}}{k_{C}}=0,36\left(\frac{D_{e} G_{C}}{\mu}\right)^{0,55}\left(\frac{C_{p} \mu}{k_{C}}\right)^{1 / 3}\left(\frac{\mu}{\mu_{P}}\right)^{0,14}(3)$
Tinker utiliza un número de Reynolds modificado con factores de corrección, para considerar el flujo radial y axial a lo largo de la coraza, dado por:

$$
\operatorname{Re}_{\mathrm{h}}=\frac{\mathrm{G}_{\mathrm{C}} \cdot \mathrm{d}}{\mu} \frac{\mathrm{F}_{\mathrm{h}}}{\mathrm{M}_{1}}
$$

Luego, correlaciona este número con el coeficiente de transferencia de calor convectivo, conforme a la ecuación 5 , a través de diagramas obtenidos a partir de diferentes arreglos de los tubos, presentados por Fraas (1989).

$$
\frac{h_{C} D_{e}}{12 k_{C}}=\left(\frac{C_{p} \mu}{k_{C}}\right)^{-1 / 3}\left(\frac{\mu}{\mu_{P}}\right)^{-0,14}
$$

En el cálculo del coeficiente global de transferencia de calor, para ambos métodos, se utiliza la siguiente expresión:

$$
\mathrm{U}_{\mathrm{c}}=\frac{\mathrm{h}_{\mathrm{i}} \mathrm{h}_{\mathrm{c}}}{\mathrm{h}_{\mathrm{i}}+\mathrm{h}_{\mathrm{c}}}
$$

Pudiendo así, a partir de las ecuaciones 1 y 6 , calcular la resistencia a la transmisión de calor total del intercambiador por la ecuación 7:

$$
R_{d}=\frac{U_{C}-U_{D}}{U_{C} U_{D}}
$$

\section{Cálculo de la pérdida de carga}

El cálculo de la pérdida de carga es realizado separadamente para el lado de la carcaza y el interior de los tubos. Para Tinker la pérdida de carga total es la suma de la pérdida de la tubería recta y de los retornos, dada por la ecuación 8:

$$
\Delta \mathrm{P}_{\mathrm{Ti}}=\Delta \mathrm{P}_{\mathrm{ti}}+\Delta \mathrm{P}_{\mathrm{ri}}
$$


Donde:

$$
\begin{array}{r}
\Delta P_{\mathrm{ti}}=\frac{\mathrm{f}_{\mathrm{i}} \cdot \mathrm{L} \cdot \mathrm{N} \cdot \mathrm{G}_{\mathrm{i}}{ }^{2}}{2 \cdot \mathrm{d}_{\mathrm{i}} \cdot \rho_{\mathrm{i}}} \\
\Delta \mathrm{P}_{\mathrm{ri}}=\frac{4 \cdot \mathrm{N} \cdot \mathrm{G}_{\mathrm{i}}{ }^{2}}{2 \cdot \rho_{\mathrm{i}}}
\end{array}
$$

Para el lado de la carcaza los méto dos de Kern y Tinker, una vez más se diferencian:

En el método de Kern el diámetro equivalente utilizado para el cálculo de la caída de presión es el mismo que el usado para la transmisión de calor, siendo la fricción adicional de la carcaza despreciada. La ecuación 11 muestra la pérdida de carga, incluyendo las pérdidas en la entrada y en la salida:

$$
\begin{gathered}
\Delta P_{C}=\frac{f_{C} G_{C}{ }^{2} D_{C}(B+1)}{2 \rho_{C} D_{e}\left(\frac{\mu}{\mu_{P}}\right)} \quad(11) \quad \begin{array}{l}
\text { siguiente ecuación: } \\
\Delta P_{C}=\frac{f_{C}\left(F_{p} \cdot G_{C}\right)^{2} x^{0,5}(B+1)}{2 \cdot \rho_{C} \cdot C_{m}}\left[1,075\left(1-\frac{H}{D_{C}}\right)\right] \cdot\left(1+Y \frac{S}{D_{C}}\right) \cdot\left(\frac{\mu_{P}}{\mu}\right)^{0,14}
\end{array}
\end{gathered}
$$

En la ecuación 10 el factor de fricción $\mathrm{f}$ es obtenido a través de un diagrama experimental, desarrollado por Kern, que lo relaciona con el número de Reynolds.

Tinker, utiliza un número de Reynolds modificado para la caída de presión, donde es considerado el flujo radial y axial a lo largo de la coraza, la fricción adicional causada por el contacto del fluido, los deflectores y partes internas de la coraza:

$$
\operatorname{Re}_{p}=\frac{F_{p} \cdot G_{c} \cdot d}{12 \cdot \mu}
$$

Luego, este número es correlacionado con el factor de fricción a través de diagramas obtenidos a partir de diferentes arreglos de los tubos, presentados por Fraas (1989). La pérdida de carga es obtenida a través de la

\section{RESULTADOS Y DISCUSION}

El intercambiador de calor fue proyectado para calentar $7000 \mathrm{~kg} \mathrm{~h}^{-1}$ de jugo de naranja de 50 a $60^{\circ} \mathrm{C}$. Para esto, fueron utilizados $20000 \mathrm{~kg} \mathrm{~h}^{-1}$ de agua a $90^{\circ} \mathrm{C}$ como fluido calefactor.
Realizando el balance de calor aplicando la ecuación 1 para el jugo, se obtiene:

$Q=68255,8 \mathrm{~J} / \mathrm{s}$

Igualando el calor intercambiado, la temperatura de salida del agua es:

$\mathrm{T}_{2}=87,2^{\circ} \mathrm{C}$ 


\section{Propiedades físicas}

La tabla 1 muestra las propiedades físicas del jugo de naranja concentrado de $27^{\circ} \mathrm{Brix}$ y del agua evaluadas a la temperatura media de cada fluido, según Telis-Romero (1998, 1999):

Tabla 1. Propiedades físicas del jugo de naranja $\left(27^{\circ} \mathrm{Brix}\right)$ a $55^{\circ} \mathrm{C}$ y agua a $88,6^{\circ} \mathrm{C}$

\begin{tabular}{llrr}
\hline & & Jugo de naranja & \multicolumn{1}{c}{ Agua } \\
\hline $\mathbf{C}_{\mathbf{p}}$ & $\left(\mathrm{J} \cdot \mathrm{Kg}^{-1} \cdot \mathrm{K}^{-1}\right)$ & 3510.30 & 4314.20 \\
$\mathbf{k}$ & $\left(\mathrm{W} \cdot \mathrm{m}^{-1} \cdot \mathrm{K}^{-1}\right)$ & 0.49 & 0.65 \\
$\mathbf{r}$ & $\left(\mathrm{Kg} \cdot \mathrm{m}^{-3}\right)$ & 1087.70 & 953.10 \\
$\mathbf{t}_{\mathbf{0}}$ & $(\mathrm{Pa})$ & 0.20 & -- \\
$\mathbf{K}$ & $\left(\mathrm{Pa} \cdot \mathrm{s}^{\mathrm{n}}\right)$ & 0.21 & -- \\
$\mathbf{n}$ & -- & 0.68 & -- \\
$\mathbf{m}$ & $(\mathrm{Pa} . \mathrm{s})$ & -- & $3.110^{-4}$ \\
\hline
\end{tabular}

Fuente: Telis-Romero et al., (1998) y Telis-Romero et al., (1999).

El análisis termohidráulico fue realizado para un intercambiador de calor con las siguientes características:

Intercambiador $1: 2$

Arreglo Triangular de los Tubos

$\mathrm{S}=0,03175 \mathrm{~m}$

$\mathrm{B}=14$

$D_{c}=19,25$ in

$\mathrm{L}=2,5 \mathrm{~m}$

Tubos: BWG 16

$X=152$ tubos

$d=1$ in

$d_{i}=0,87$ in

\section{Proyecto térmico}

Ambos métodos son iguales para el cálculo del proyecto térmico en el interior de los tubos, siendo los datos presentados a continuación:

$$
\begin{array}{lll}
A_{i} & =0,0289 & m^{2} \\
G_{i} & =67,33 & \mathrm{Kg} \cdot \mathrm{m}^{-2} \cdot \mathrm{s}^{-1} \\
\mathrm{Re}_{i}=17,79 & \\
\mathrm{~h}_{i} & =174,74 & \mathrm{~W} \cdot \mathrm{m}^{-1} \cdot \mathrm{K}^{-1}
\end{array}
$$

Los resultados de los proyectos térmicos del lado

\begin{tabular}{|c|c|}
\hline Kern & Tinker \\
\hline$A_{c}=0.163 \mathrm{~m}^{2}$ & $A_{c}=0.0163 \mathrm{~m}^{2}$ \\
\hline $\mathrm{G}_{\mathrm{c}}=340.87 \mathrm{Kgm}^{-2} \mathrm{~s}^{-1}$ & $\mathrm{G}_{\mathrm{c}}=340.87 \mathrm{Kg} \mathrm{m}^{-2} \mathrm{~s}^{-1}$ \\
\hline $\mathrm{Re}_{c}=19856.61$ & $\operatorname{Re}_{h}=8569$ \\
\hline $\mathrm{h}_{\mathrm{c}}=22513.81 \mathrm{~W} \mathrm{~m}^{-1} \mathrm{~K}^{-1}$ & $\mathrm{~h}_{\mathrm{c}}=31964.46 \mathrm{~W} \mathrm{~m}^{-1} \mathrm{~K}^{-1}$ \\
\hline $\mathrm{U}_{c}=173.34 \mathrm{~W} \mathrm{~m}^{-1} \mathrm{~K}^{-1}$ & $\mathrm{U}_{c}=173.79 \mathrm{~W} \mathrm{~m}^{-1} \mathrm{~K}^{-1}$ \\
\hline$U_{D}=77.71 \mathrm{~W} \mathrm{~m}^{-1} \mathrm{~K}^{-1}$ & $\mathrm{U}_{\mathrm{D}}=77.71 \mathrm{~W} \mathrm{~m}^{-1} \mathrm{~K}^{-1}$ \\
\hline$R_{d}=0.0071 \mathrm{mKW}^{-1}$ & $R_{d}=0.0071 \mathrm{mKW}^{-1}$ \\
\hline
\end{tabular}
de la coraza para los métodos de Kern y Tinker se muestran en la tabla 2.

Tabla 2. Resultados del proyecto térmico del lado de la carcaza 


\section{Proyecto hidráulico}

Los métodos en cuestión son iguales para el cálculo del proyecto hidráulico en el interior de los tubos, siendo los datos presentados a continuación:

$\begin{array}{rlr}f_{i} & =3,60 & \\ D P_{t i} & =12938,80 & \mathrm{~Pa} \\ D P_{r i} & =16,73 & \mathrm{~Pa} \\ D P_{T i} & =12955,53 & \mathrm{~Pa}\end{array}$

Los resultados de los proyectos hidráulicos del lado de la carcaza para los métodos de Kern y Tinker se presentan en la tabla 3.

Como se observa en la tabla 1 el jugo de naranja presenta un comportamiento de un plástico de Casson; como no hay correlación para el cálculo del coeficiente de transferencia de calor por convección para este tipo de fluido, se utiliza la ecuación 2, aplicada para fluidos que cumplen ley de potencia, lo que no causa errores significativos, pues el valor de $t_{0}$ es pequeño.

Los dos métodos se diferencian en los cálculos termohidráulicos solamente para el flujo por el lado de la carcaza. En cuanto Kern desarrolla su método apenas para flujo radial, Tinker considera flujo tanto en la dirección radial como axial, lo que genera diferentes coeficientes de transferencia de calor (tabla 2). Estas desigualdades podrían causar mayores errores en el intercambiador proyectado por el método de Kern, siendo el método de Tinker el más exacto, por considerar condiciones de contorno próximas a la real.

Los proyectos en cuestión no presentan diferencias en cuanto a las resistencias térmicas, debido a que el fluido en el interior de los tubos presenta un flujo laminar, que lleva a una mayor resistencia térmica en relación a la coraza (alrededor de $1500 \%$ ), que a su vez, desarrolla un flujo turbulento. Además, las conductividades térmicas y las propiedades reológicas de los fluidos, mostradas en la tabla 1, favorecen un mayor intercambio de calor del lado de la carcaza.

Las pérdidas de carga calculadas por ambos métodos se muestran en la tabla 3. Las diferencias en los valores se explican por el hecho de Tinker considerar un área de pérdida de carga mayor, ocasionando un flujo másico y número de Reynolds menores.

Debido a que el método de Tinker es considerado el más exacto, induciría a un super dimensionamento en el proyecto hidráulico del orden de $300 \%$.

Tabla 3. Resultados del proyecto hidráulico del lado de la carcaza

\begin{tabular}{ll}
\hline \multicolumn{1}{c}{ Kern } & \multicolumn{1}{c}{ Tinker } \\
\hline $\operatorname{Re}_{C}=19856,61$ & $\operatorname{Re}_{p}=13084,5$ \\
$\mathrm{f}_{C}=3,60$ & $\mathrm{f}_{C}=0,51$ \\
$D P_{C}=7226,72 \mathrm{~Pa}$ & $\mathrm{DP}_{C}=2083,00 \mathrm{~Pa}$ \\
\hline
\end{tabular}




\section{CONCLUSIONES}

- Los coeficientes de transferencia de calor convectivos, para la coraza calculados por los métodos estudiados presentaron diferencias significativas del orden de $70 \%$.

- Las resistencias térmicas fueron exactamente iguales debido a una mayor resistencia térmica encontrada en el interior de los tubos donde los métodos no se diferencian.

- En el dimensionamento hidráulico las pérdidas de carga encontradas difieren alrededor de $300 \%$.

- El mismo intercambiador dimensionado por los dos métodos presentará diferentes especificaciones.

\section{BIBLIO G RAFIA}

Fraas, A. 1989. Heat Exchanger Design. John Wiley and Sons Inc., New York, p.228-245

Geankoplis, C. 1993. Transport process and unit operations. Englewood Cliffs, Prentice-Hall, N ew York, p.297

Kern, D. 1999. Procesos de Transmisión de Calor. McGraw Hill Book Company, Inc. M éxico DF, p159-512

O zisik, M. 1990. Transferencia de Calor - U m texto básico. Guanabara Koogan S.A. Río de Janeiro, p.447-502
Telis-Romero, J.; Telis, V.; Gabas, A. y Yamashita F. 1998. Thermophysical properties of Brazilian orange juice as affected by temperature and water content. Journal of Food Engineering 38:27-40

Telis-Romero, J.; Telis, V. y Yamashita, F. 1999. Friction factors and rheological properties of orange juice. Journal Food Engineering 40:101-106 